\title{
A NOTE CONCERNING HYPERBOLIC EQUATIONS WITH CONSTANT COEFFICIENTS*
}

By JAMES F. HEYDA (Aircraft Nuclear Propulsion Department, General Electric Co.)

The canonical form of the linear, homogeneous hyperbolic partial differential equation in two independent variables is

$$
u_{x y}+a u_{x}+b u_{y}+c u=0,
$$

where $a, b, c$ are functions of $x, y$. The constant coefficients case, frequently met in applitions, can be reduced to the simpler form

$$
v_{x y}-\lambda v=0, \quad \lambda=a b-c
$$

with $\lambda$ constant, through the substitution

$$
v(x, y)=u \exp (b x+a y) .
$$

The solution of the Cauchy problem for an equivalent form of (2) with $v$ and $\partial v / \partial n$ prescribed along $C$ is worked out explicitly by Copson [1] using the methods of M. Riesz.

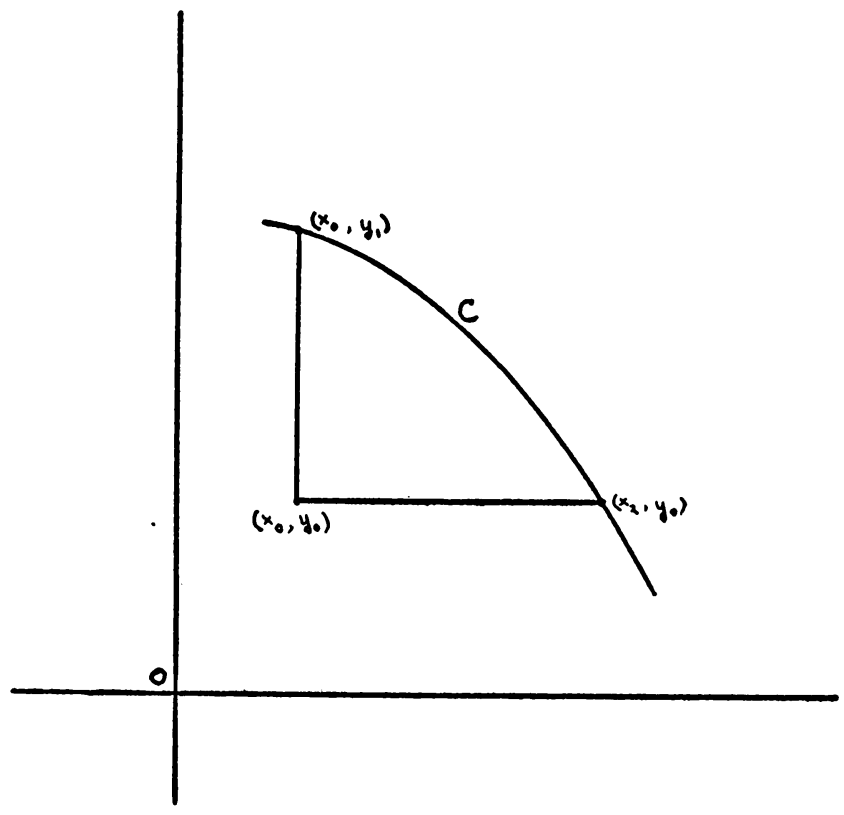

FIG. 1

The form of Copson's solution is the same as that which one would obtain by using Riemann's method for solving the same problem. This enables us to identify the Bessel function $I_{0}\left(2[\lambda(x-\alpha)(y-\beta)]^{1 / 2}\right)$ as the Riemann characteristic function for Eq. (2) By a theorem of Le Roux [2], it then follows that the definite integrals

${ }^{*}$ Received September 19, 1958; revised manuscript received August 28, 1959. 


$$
\begin{aligned}
& \int_{x_{0}}^{x} f(\alpha) I_{0}\left(2\left[\lambda\left(y-y_{0}\right)(x-\alpha)\right]^{1 / 2}\right) d \alpha, \\
& \int_{y_{0}}^{y^{2}} g(\beta) I_{0}\left(2\left[\lambda\left(x-x_{0}\right)(y-\beta)\right]^{1 / 2}\right) d \beta
\end{aligned}
$$

will also be solutions of (2) for arbitrary functions $f(\alpha)$ and $g(\beta)$. This enables us to write down directly a solution of (2) for prescribed values of $v(x, y)$ along the characteristic lines $x=x_{0}$ and $y=y_{0}$. For we have from (4) by superposition

$$
\begin{aligned}
& v(x, y)=\int_{x_{0}}^{x} f(\alpha) I_{0}\left(2\left[\lambda\left(y-y_{0}\right)(x-\alpha)\right]^{1 / 2}\right) d \alpha \\
& \quad+\int_{y_{0}}^{y} g(\beta) I_{0}\left(2\left[\lambda\left(x-x_{0}\right)(y-\beta)\right]^{1 / 2}\right) d \beta+v\left(x_{0}, y_{0}\right) I_{0}\left(2\left[\lambda\left(x-x_{0}\right)\left(y-y_{0}\right)\right]^{1 / 2}\right),
\end{aligned}
$$

where

$$
f(x)=\frac{\partial v\left(x, y_{0}\right)}{\partial x}, \quad g(y)=\frac{\partial v\left(x_{0}, y\right)}{\partial y},
$$

the prescribed functions $v\left(x, y_{0}\right), v\left(x_{0}, y\right)$ being assumed suitably well-behaved to carry out the operations indicated.

As an instance of (5) we remark that it furnishes directly a closed form solution of the boundary value problem treated by Mason [3] in connection with heat transfer in cross-flow and explains the mysterious appearance of $I_{0}\left(2[a b x y]^{\frac{1}{2}}\right)$ in his solution, arrived at by means of Laplace transform methods.

\section{ReFerenCes}

1. E. T. Copson, Some applications of Riesz's method, Proceedings of the Conference on Differential Equations, University of Maryland Press, 1956, p. 113

2. J. B. Diaz and G. S. S. Ludford, On the Euler-Poisson-Darboux equation, Ibid., p. 82

3. J. L. Mason, Heat transfer in cross-flow, Proceedings of the Second U. S. National Congress of Applied Mechanics, 1954, p. 801

Corrections to the paper

THERMAL INSTABILITY OF VISCOUS FLUIDS

Quarterly of Applied Mathematics, XVII, 25-42 (1959)

BY CHIA-SHUN YIH (University of Michigan)

Equation (27) should read

$$
\left[\sigma-\operatorname{Pr}\left(D^{2}-b^{2}-a^{2}\right)\right]\left(D^{2}-a^{2}\right) f=-R \operatorname{Pr} D \theta
$$

The second sentence following Eq. (27) should then be changed to: "Since Eqs. (26) and (27) would be identical to Eqs. (20) and (21) if $\sigma+b^{2}$ is replaced by $\sigma$, any variation of the flow with $y$ will invariably make the flow more stable, if $u_{2}$ remains strictly zero, and the boundary conditions on $f$ itself are not relaxed."

With the periodic variation in the $y$-direction provided, the boundary conditions 